\title{
Bubble Chambers for Experiments in Nuclear Astrophysics
}

\author{
B. DiGiovine \\ Physics Division, Argonne National Laboratory, Argonne, IL 60439, USA \\ D. Henderson \\ Physics Division, Argonne National Laboratory, Argonne, IL 60439, USA \\ R. J. Holt \\ Physics Division, Argonne National Laboratory, Argonne, IL 60439, USA \\ R. Raut \\ UGC-DAE Consortium for Scientific Research, Kolkata Centre LB-8 Sector-III \\ Bidhannagar, Kolkata 700098, India.
}

K. E. Rehm

Physics Division, Argonne National Laboratory, Argonne, IL 60439, USA

A. Robinson

Department of Physics, University of Chicago, Chicago, IL 60637, USA

A. Sonnenschein

Fermi National Accelerator Laboratory, Batavia, IL 60510, USA

G. Rusev

Chemistry Division, Los Alamos National Laboratory, Los Alamos, New Mexico 87545, USA

A. P. Tonchev

Physics Division, Lawrence Livermore National Laboratory, Livermore, California 94550, $U S A$

C. Ugalde $^{1}$

Department of Physics, University of Illinois at Chicago, Chicago, IL 60607, USA

\footnotetext{
${ }^{1}$ email address: cugalde@anl.gov
} 


\section{${ }_{26}$ Abstract}

27 A bubble chamber has been developed to be used as an active target system for low energy nuclear astrophysics experiments. Adopting ideas from dark matter detection with superheated liquids, a detector system compatible with $\gamma$-ray beams has been developed. This detector alleviates some of the limitations encountered in standard measurements of the minute cross sections of interest to stellar environments. While the astrophysically relevant nuclear reaction processes at hydrostatic burning temperatures are dominated by radiative captures, in this experimental scheme we measure the time-reversed processes. Such photodisintegrations allow us to compute the radiative capture cross sections when transitions to excited states of the reaction products are negligible. Due to the transformation of phase space, the photodisintegration cross sections are up to two orders of magnitude higher. The main advantage of the new target-detector system is a density several orders of magnitude higher than conventional gas targets. Also, the detector is virtually insensitive to the $\gamma$-ray beam itself, thus allowing us to detect only the products of the nuclear reaction of interest. The development and the operation as well as the advantages and disadvantages of the bubble chamber are discussed.

\section{Introduction}

All elements of the periodic table with $\mathrm{Z}>3$ have been produced through nuclear reactions in the interior of stars via quiescent or explosive burning processes. The cross sections of these reactions however, are very small (typically $\mathrm{pb}-\mathrm{fb}$ ) so that they are difficult to measure even at the high temperatures and energies occurring in stellar explosions. In stellar environments this is compensated by the large masses and long time scales involved in stellar evolution. The thin targets which must be used in terrestrial reaction studies (typically $\mu \mathrm{g}-\mathrm{mg} / \mathrm{cm}^{2}$ ) result in very small luminosities and count rates, with the result that up to now only very few astrophysical reaction cross sections have been measured at stellar temperatures. Most of the other reactions are studied at 
higher energies and the cross sections are then extrapolated towards the astrophysical energies of interest. In this paper we describe a new detector system working with active liquid targets, which for radiative capture reactions gives an increase in luminosity by several orders of magnitude. Together with existing and planned new accelerators this may enable measurements of many reactions of astrophysical interest under stellar conditions.

\section{Astrophysical background}

The radiative capture of hydrogen or helium (i.e. $(\mathrm{p}, \gamma)$ or $(\alpha, \gamma)$ reactions) on light nuclei such as carbon, nitrogen and oxygen are some of the most important processes in stellar nucleosynthesis. These capture reactions have been studied for many years by bombarding targets of carbon, nitrogen or oxygen with intense proton and $\alpha$-particle beams or (in so-called inverse kinematics) by bombarding hydrogen and helium gas targets with heavier particles at energies of a few hundreds of $\mathrm{keV} / \mathrm{u}$ and detecting the reaction products with suitable detectors. The thin targets $\left(\sim 10 \mu \mathrm{g} / \mathrm{cm}^{2}\right)$ which are required at these energies together with the beam intensities available at present particle accelerators result in low luminosities with count rates that reach typically 1 count/day for cross sections in the pb region. Small improvements of these yields are still possible but quite long running times will nonetheless be required. If the reaction products from e.g. an $(\alpha, \gamma)$ reaction are stable, a considerable improvement of the luminosity can be achieved by studying the inverse $(\gamma, \alpha)$ process. One improvement in the expected count rate comes from the reciprocity theorem for nuclear reactions which relates the $(\gamma, \alpha)$ process to its inverse $(\alpha, \gamma)$ reaction [1]. The two cross sections are related via

$$
\frac{\sigma(\gamma, \alpha)}{\sigma(\alpha, \gamma)}=\frac{\omega_{\alpha, \gamma} k_{\alpha, \gamma}^{2}}{\omega_{\gamma, \alpha} k_{\gamma, \alpha}^{2}},
$$

where $\mathrm{k}_{\alpha, \gamma}$ and $\mathrm{k}_{\gamma, \alpha}$ are the wave numbers for capture and photodisintegration channels, respectively, and $\omega_{\alpha, \gamma}$ and $\omega_{\gamma, \alpha}$ are the associated spin multiplicity factors. In the energy range discussed in this paper, the ratio can provide a 
gain of approximately two orders of magnitude in cross section. Another gain in luminosity is obtained from the choice of the target. At the corresponding $\gamma$-ray energies for $(\gamma, \alpha)$ reactions of $5-10 \mathrm{MeV}$ the large range of the incident $\gamma$-rays allows us to use targets with thicknesses of $\sim 1-10 \mathrm{~g} / \mathrm{cm}^{2}$, which corresponds to a factor of $10^{5-6}$ improvement in luminosity. Disadvantages of this method include the limitation of present tunable $\gamma$-ray sources to about $10^{8} \gamma / \mathrm{s}$ and the need for a detector that is insensitive to the incident $\gamma$-rays. The latter has been achieved by the use of superheated liquids in a bubble chamber to detect and measure the reaction products from the $(\gamma, \alpha)$ reaction since these detectors have a high insensitivity to $\gamma$-rays. This has been tested to a level of less than $2 \times 10^{-10}[2]$.

One of the most important capture reactions in nuclear astrophysics is the ${ }^{12} \mathrm{C}(\alpha, \gamma){ }^{16} \mathrm{O}$ reaction, sometimes called the "holy grail of nuclear astrophysics", which can be studied with this technique by using a superheated liquid active target bubble chamber operating with an oxygen-containing liquid. While a study of this reaction is planned for the future, we describe in this paper a study of the capture reaction ${ }^{15} \mathrm{~N}(\alpha, \gamma){ }^{19} \mathrm{~F}$ via the photodissociation reaction ${ }^{19} \mathrm{~F}(\gamma, \alpha){ }^{15} \mathrm{~N}$ using fluorine containing liquids. This reaction is of interest to nuclear astrophysics since fluorine is the least abundant element in the mass range between 11 and 32 as shown by its solar abundance. This suggests that either it is very hard to synthesize or extremely fragile in stellar environments. Various scenarios for the nucleosynthesis of fluorine have been proposed. One includes the neutrino dissociation of ${ }^{20} \mathrm{Ne}$ in core collapse supernovae [4]. Others suggest that ${ }^{19} \mathrm{~F}$ could be produced both during the thermal pulse phase in the intershell region of Asymptotic Giant Branch (AGB) stars. Another possibility includes hydrostatic burning in the helium shell of Wolf-Rayet stars. In both cases, the nuclear reaction sequence is the same, with the exception that in the AGB star case, the required neutron flux is induced by ${ }^{13} \mathrm{C}$ nucleosynthesis produced by the mixing of hydrogen from the envelope into the intershell region and captured by the increasingly abundant ${ }^{12} \mathrm{C}$ nucleus. For the latter part cross section measurements of the ${ }^{15} \mathrm{~N}(\alpha, \gamma){ }^{19} \mathrm{~F}$ reactions at astrophysical energies are 
needed. In this contribution we describe such a measurement using this newly developed detector using the inverse photodissociation process.

\section{The Bubble Chamber for use in nuclear astrophysics experiments}

The bubble chamber makes use of the instability of superheated liquids against bubble formation for the detection of charged particles from a nuclear reaction. Detectors of this type have been exploited in high-energy physics experiments for more than 50 years when D. A. Glaser suggested their use to visualize the tracks of high-energy charged particles [5]. Later this technique also found applications in low-energy physics for neutron detection [6]. Larger variants of these detectors are currently employed in dark matter searches $[7,8,9]$.

The most important difference between "standard" bubble chambers used in high-energy physics and the new class of bubble chambers is in their mode of operation. The bubble chambers of high-energy physics were pulsed with the beam bunches, which arrived at well defined time intervals. As a result the bubble chamber would only spend a fraction of a second in the superheated state. In our application the bubble chamber must stay continuously active until a nuclear reaction occurs in the superheated liquid. With the small cross sections of interest, the active time can be minutes or hours, and so special care must be taken to prevent spurious boiling which is not caused by the nuclear reaction of interest. Another important difference between the device and experiments proposed here and those of high energy physics is that no tracks are left by the particle inducing nucleation. For the experiments relevant to nuclear astrophysics, the energies of the reaction products are so small that they are stopped in the liquid after a few microns. This means that no direct kinematic information can be obtained with this detector. Borrowing heavily from successful designs used in the search for dark matter [3, 7, 9], we have designed and tested a superheated active target system which will be described in the following sections. 


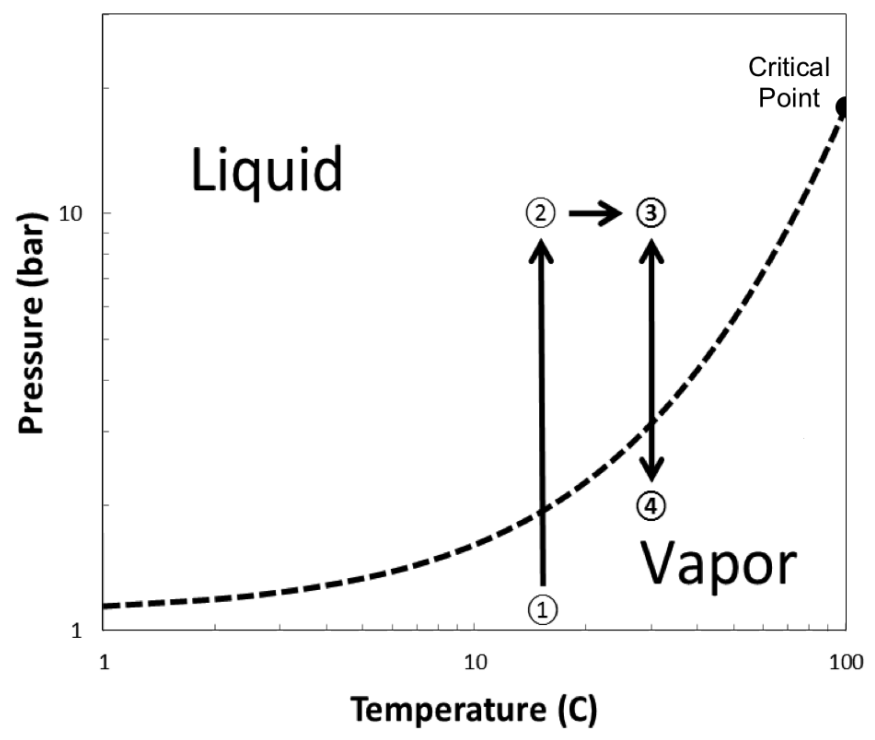

Figure 1: Phase diagram of $\mathrm{C}_{4} \mathrm{~F}_{10}$. The solid line represents the path used to bring the fluid into its superheated state

\subsection{Thermodynamics of bubble detectors}

The study of nucleation in a superheated system has a long history, and is still being investigated today $[10,11,12]$. The theory of bubble formation in a superheated liquid (the so-called "thermal spike" model) has been discussed in detail by Seitz [13] and will not be repeated here. In the following we provide only a few of the equations, which are necessary for understanding the operating principle. Figure 1 shows the phase diagram for $\mathrm{C}_{4} \mathrm{~F}_{10}$.

The thick solid lines in figure 1 indicate the path that is being used to generate a superheated liquid. Starting at a point where the material is gaseous under ambient pressure and temperature (1), the pressure is first increased, liquifying the gas (2), followed by an increase in temperature (3). Decreasing the pressure below the liquid-gas phase boundary curve brings the fluid into its superheated state (4). For $\mathrm{C}_{4} \mathrm{~F}_{10}$ the operating temperatures are near $30^{\circ} \mathrm{C}$ and the superheat pressures are typically 1-4 bar. If left undisturbed, the fluid will remain liquid in this metastable state. If it experiences a disturbance, which deposits enough energy into the liquid within a certain distance, a critical barrier 
can be overcome and a "proto-bubble" forms. If the "proto-bubble" exceeds a critical radius $R_{c}$ it becomes unstable and the phase transition continues from superheated liquid into the vapor state creating a macroscopic bubble. The critical radius is given by energy conservation arguments as

$$
R_{c}=\frac{2 \times \sigma}{\left(p_{v}-p_{e}\right)},
$$

where $\sigma$ is the (temperature dependent) surface tension of the liquid, and $\Delta \mathrm{p}=\mathrm{p}_{v}-\mathrm{p}_{e}$ the degree of superheat, i.e. the difference between the vapor pressure $\mathrm{p}_{v}$ at the operating temperature, and the fluid pressure $\mathrm{p}_{e}$. Since the surface tension decreases with temperature, the critical radius $\mathrm{R}_{c}$ decreases towards higher temperatures. Typical values of $\mathrm{R}_{c}$ for the active fluid $\mathrm{C}_{4} \mathrm{~F}_{10}$, discussed in this paper, are between tens and hundreds of $\mathrm{nm}$.

The energy $\mathrm{E}_{c}$ needed to form a bubble with a critical radius $\mathrm{R}_{c}$, is given [10] by the equation

$$
\begin{aligned}
E_{c}= & -\frac{4}{3} \pi R_{c}^{3} \Delta p+\frac{4}{3} \pi R_{c}^{3} \rho_{v} H_{l v} \\
& +4 \pi R_{c}^{2}\left(\sigma-T \frac{d \sigma}{d T}\right)+W_{i r r}
\end{aligned}
$$

In equation $(3), \rho_{v}$ is the density of the material in its gas phase, $\mathrm{H}_{l v}$ is the latent heat of evaporation of the superheated liquid and $\mathrm{W}_{i r r}$ is the energy that goes into irreversible processes such as sound. The first terms in equation (3) correspond, respectively, to the reversible work during the expansion, the energy needed to evaporate the liquid and the energy needed to generate the bubble surface of radius $\mathrm{R}_{c}$. The irreversible contributions expressed by the last term $\mathrm{W}_{\text {irr }}$ are small and are usually neglected in the calculations. The various terms contributing to $\mathrm{E}_{c}$ for $\mathrm{C}_{4} \mathrm{~F}_{10}$ are compared in Fig. 2. The values for the thermodynamic parameters were obtained from the NIST code [14]. In these calculations, it was assumed that the pressure was 1.82 bar, which corresponds to the operating value used to superheat the liquid. As can be seen from Fig. 2 , the dominant contribution to $\mathrm{E}_{c}$ comes from the energy needed to evaporate 


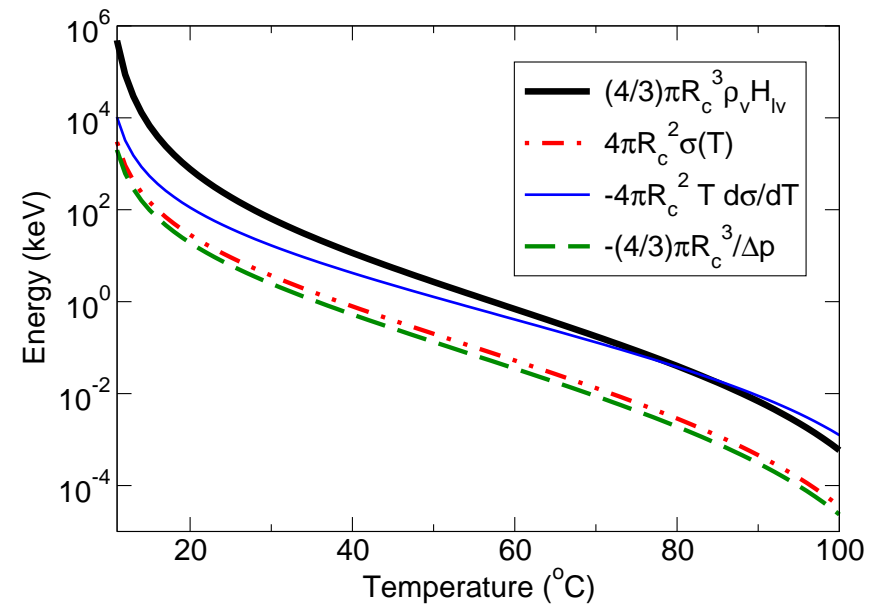

Figure 2: The four terms contributing to the critical energy necessary to form a bubble of critical radius at an operating pressure of 1.82 bar for the liquid $\mathrm{C}_{4} \mathrm{~F}_{10}$.

the liquid, followed by the term originating from the temperature dependence of the surface tension. The contributions from the work needed to generate the surface of a bubble or from the reversible work during the expansion phase are a factor of $\sim 10$ smaller.

The "thermal spike" for creating a bubble can come from two sources: (1) In the photo-dissociation reaction ${ }^{19} \mathrm{~F}(\gamma, \alpha){ }^{15} \mathrm{~N}$ two charged particles, ${ }^{15} \mathrm{~N}$ and an $\alpha$-particle are produced. Depending on the energy of the incoming $\gamma$-ray (5-6 $\mathrm{MeV}$ in the experiment described later) the energies are in the range of 200-500 $\mathrm{keV}$ for ${ }^{15} \mathrm{~N}$ and between $800-1800 \mathrm{keV}$ for the $\alpha$ particle, respectively. (2) In addition there are background reactions induced by neutrons from cosmic rays. While the liquid in the bubble chamber itself is insensitive to neutrons (neutrons themselves are unable to trigger nucleation), it can be triggered by the ${ }^{12} \mathrm{C}$ or the ${ }^{19} \mathrm{~F}$ recoils produced in an elastic collision. For a $1 \mathrm{MeV}$ neutron, these recoil energies are in the range of $\sim 300 \mathrm{keV}\left({ }^{12} \mathrm{C}\right)$ or $200 \mathrm{keV}\left({ }^{19} \mathrm{~F}\right)$, respectively, scaling with the energy of the incoming neutron.

In order to generate the thermal spike, these nuclei need to deposit this 


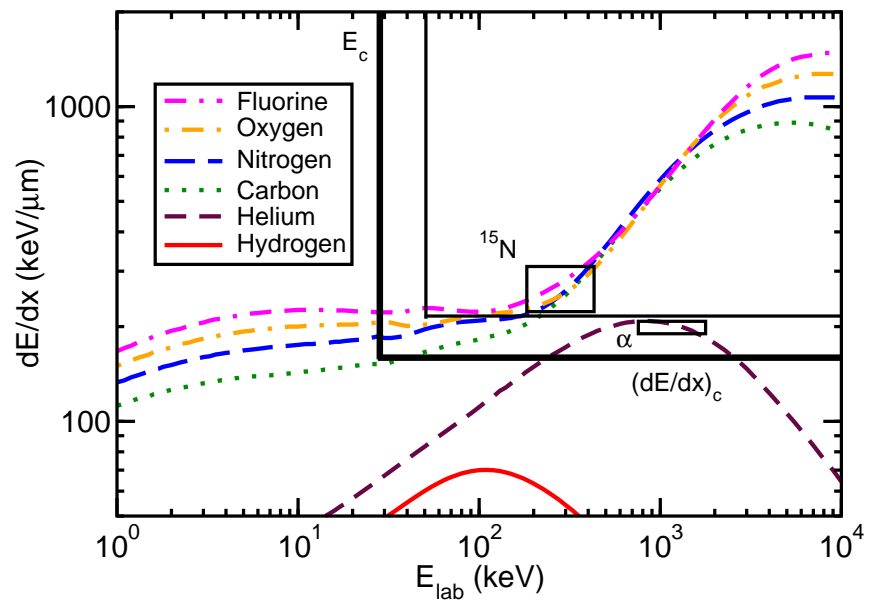

Figure 3: Detection thresholds for the bubble chamber at two different superheats (see text). The curves represent stopping powers for various nuclei moving in $\mathrm{C}_{4} \mathrm{~F}_{10}$. The liquid will nucleate (form bubbles) for particles in the region delimited by $\mathrm{dE} / \mathrm{dx}_{c}$ and $\mathrm{E}_{c}$, which are a function of the superheat in the liquid. These are dependent on the pressure and temperature of the liquid. Typical stopping powers and kinetic energies for the ${ }^{19} \mathrm{~F}(\gamma, \alpha){ }^{15} \mathrm{~N}$ experiment described in this paper are shown by the small squares for $\alpha$-particles and ${ }^{15} \mathrm{~N}$. The superheats used for the experiment must define detection thresholds that include the kinematics of the particles as represented in the small squares. When a photodisintegration event produces recoils within the thresholds, a visible bubble will be produced and detected with the CMOS cameras.

energy over a suitably short distance. This distance is somewhat debated in the literature [15] but typical values range from $2-6 \times R_{c}$.

If we integrate the energy-loss curves of the charged particles mentioned above over a typical range of $2-6 \times R_{c}$ we obtain the deposited energy $\mathrm{E}_{d e p}$ which needs to be larger than $\mathrm{E}_{c}$ in order to initiate a macroscopic bubble. These conditions allow us to select the sensitivity thresholds for particle detection by choosing the proper temperatures and pressures (superheat) in the bubble chamber. In Fig. 3 the stopping power curves for several charged particles from hydrogen to ${ }^{19} \mathrm{~F}$ in $\mathrm{C}_{4} \mathrm{~F}_{10}$ are shown as a function of the energy. Also included are the detection limits in $\mathrm{dE} / \mathrm{dx}$ and $\mathrm{E}$ for operating the bubble chamber at $\mathrm{T}=33{ }^{\circ} \mathrm{C}$ and $\mathrm{P}=1.82$ bar, respectively (thick solid lines). As can be seen at these pressures, the bubble chamber is insensitive to protons. The detector can also 


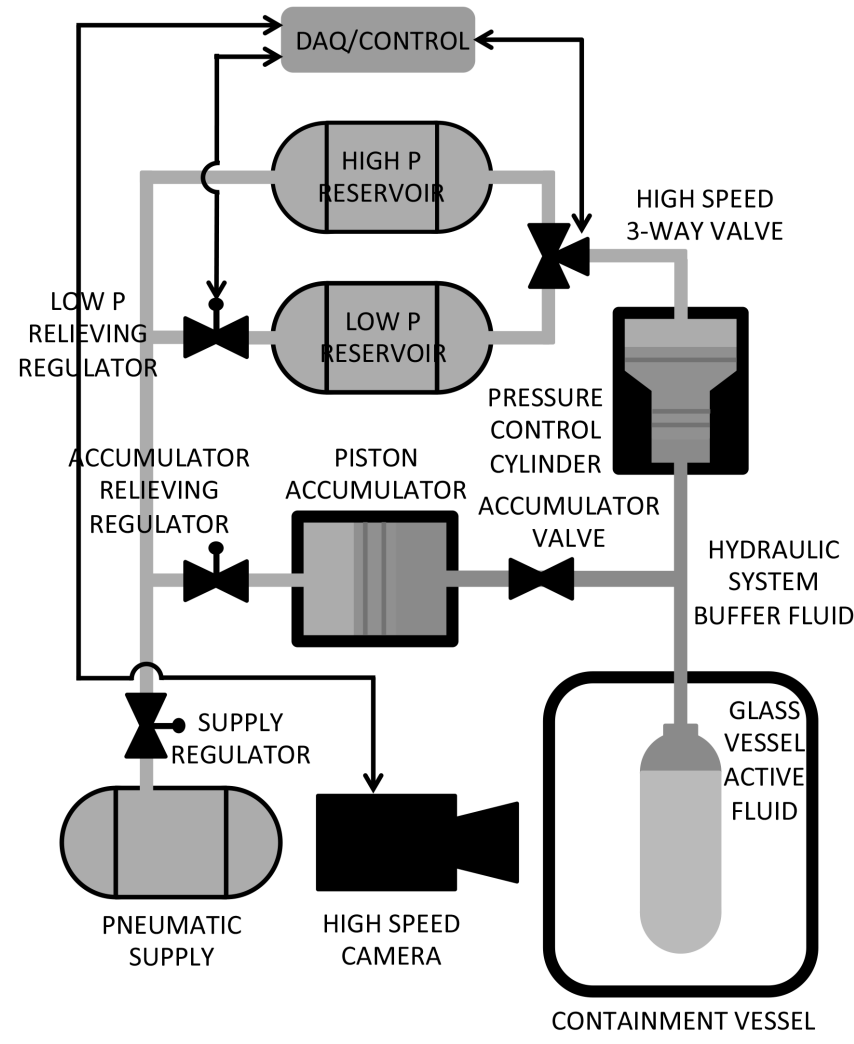

Figure 4: Schematic of the superheated liquid active target system, showing the main subsystems of the apparatus.

be made insensitive to $\alpha$-particles by choosing a smaller amount of superheat, as shown by the thin solid lines, which correspond to operating conditions of $\mathrm{T}=30{ }^{\circ} \mathrm{C}$ and $\mathrm{P}=1.82$ bar.

\section{Mechanical details}

Figure 4 gives an overall schematic of the basic sub-systems of the bubble chamber. The containment vessel is filled with the active target fluid (bottom) and a so-called buffer fluid (top) fill the volume of the hydraulic system. The active fluid resides in a high pressure glass vessel allowing the less dense buffer fluid to fill the rest of the hydraulic system. A piston accumulator compen- 
sates for volumetric changes during filling and temperature adjustments of the active fluid. The hydraulic system pressure is controlled via a pneumatically driven pressure control cylinder assembly. The pneumatic system has a highand low-pressure reservoir, which are connected to the pressure control cylinder via a high-speed three way valve. The low- pressure reservoir is fed by a precision relieving regulator and sets the superheat pressure of the system. The high-pressure reservoir is fed directly from the regulated pneumatic supply and sets the compression pressure, which is typically a factor of $2-3$ higher than the vapor pressure of the fluid at operating temperature. The glass pressure vessel resides within a containment vessel which protects operators and equipment in the event of glass failure. High speed CMOS cameras operated by a data acquisition and control (DAQ\&C) system continuously monitor the active volume taking pictures every $10 \mathrm{~ms}$, analyzing and capturing events in the superheated fluid. The detection of an event is achieved by comparing successive pictures: subtracting the individual pixels from one image to the next. Upon event detection the system is repressurized within $60 \mathrm{~ms}$, which liquifies the gas bubble and resets the system, allowing for subsequent depressurization back to the superheated state. A compression event is shown in figure 5 .

The bubble chamber was operated for several weeks before the experimental campaigns were started. Cyclic fatigue was not observed as the device was subjected to pressure changes that induced the superheat in the liquid.

\subsection{Active target and buffer fluid}

Since in our application the bubble chamber must stay active for extended periods of time, extraneous bubble formation must be kept to a minimum. The active target fluid $\left(\mathrm{C}_{4} \mathrm{~F}_{10}\right)$ [16] has to be kept clean and must only contact very smooth surfaces. The fluid is therefore only allowed to come into contact with the glass pressure vessel or with the buffer fluid which provides a smooth interface for the transmission of pressure changes from the hydraulic system. The choice of the buffer fluid depends on several criteria. (1) It must have a lower density so that it floats on top of the active fluid. (2) It must be immiscible 


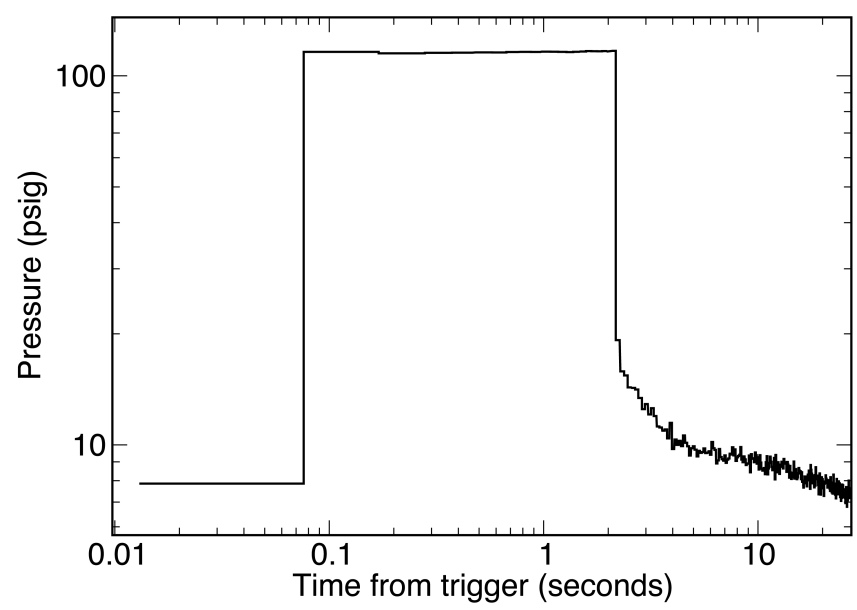

Figure 5: Compression cycle of the liquid after bubble detection. The width of the pressurized state is about two seconds, corresponding to the time it took to liquify the bubble and stabilize the liquid before the superheat was induced again.

with the active fluid in order for a meniscus to form. (3) Solubility between the active fluid and the buffer fluid must be very low, and (4) it should not become superheated in the pressure/temperature range chosen for the experiment. For $\mathrm{C}_{4} \mathrm{~F}_{10}$ as an active fluid, water turns out to be an ideal buffer fluid and was utilized in this experiment.

\subsection{Glass pressure vessel}

The active fluid is contained within a cylindrical glass vessel [17] with an overall length of $102 \mathrm{~mm}$, an inner diameter of $30 \mathrm{~mm}$, and an outer diameter of $36 \mathrm{~mm}$. The connection to the hydraulic system is provided by a threaded Oring sealed bushing. In order to determine the maximum pressure at which this vessel could be operated, several studies were performed. A three-dimensional solid model of the vessel was created using a 3D CAD software package. This model was then used to perform stress analysis simulations, which predicted a failure of the vessel at pressures around 100 bar. Hydrostatic tests were then performed up to 80 bar on several vessels, with no failures observed. Considering simulations and hydrostatic testing, the glass vessel provided our system with a safety factor of about 8-10. Because of the brittle nature of glass and the 


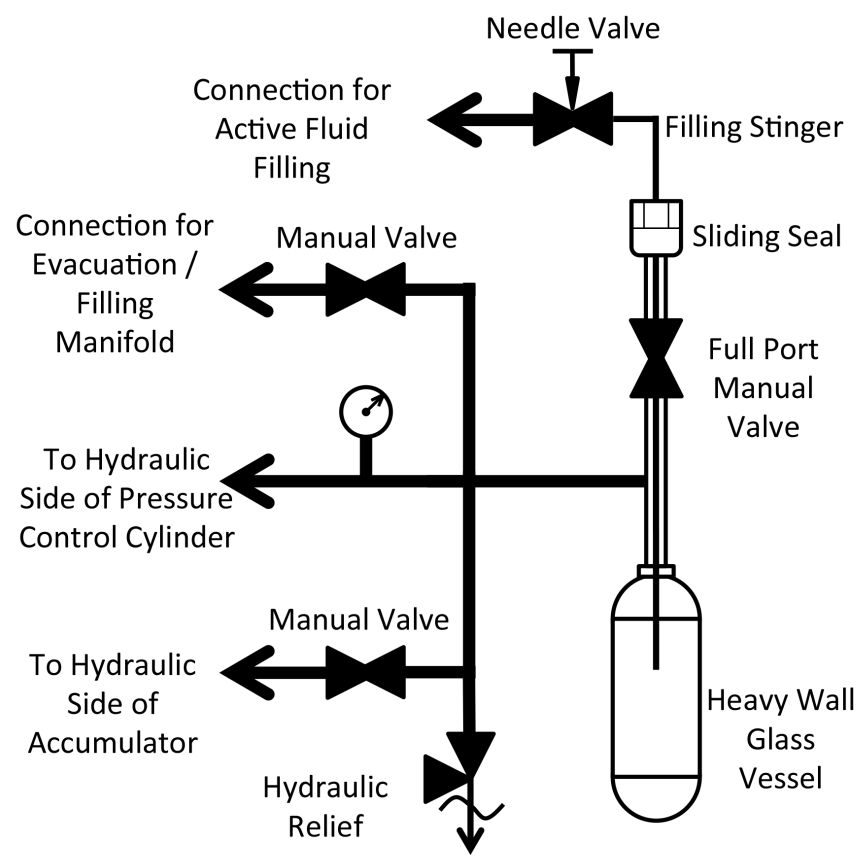

Figure 6: Schematic of the hydraulic system.

possibility of stress risers, which can occur in glass due to small scratches or imperfections, the glass vessel was housed within a secondary stainless steel containment vessel.

\subsection{Hydraulic System}

There are several requirements for the hydraulic system. (1) It provides the interface between the superheated liquid and the pneumatic pressure control system, (2) it allows for thermal expansion compensation, and (3) enables the filling of the pressure vessel with the active fluid. A schematic diagram is shown in Fig. 6. The hydraulic system pressure is controlled by utilizing an unbalanced piston and cylinder assembly which is the main interface between the hydraulic and the pneumatic system. The design of this piston assembly is shown in Fig. 7. The hydraulic side of the cylinder has a diameter of about $40 \%$ of the pneumatic side, allowing for higher pressures to be reached using standard off-the-shelf pneumatic control valves, regulators and tanks. The travel of the piston in both 


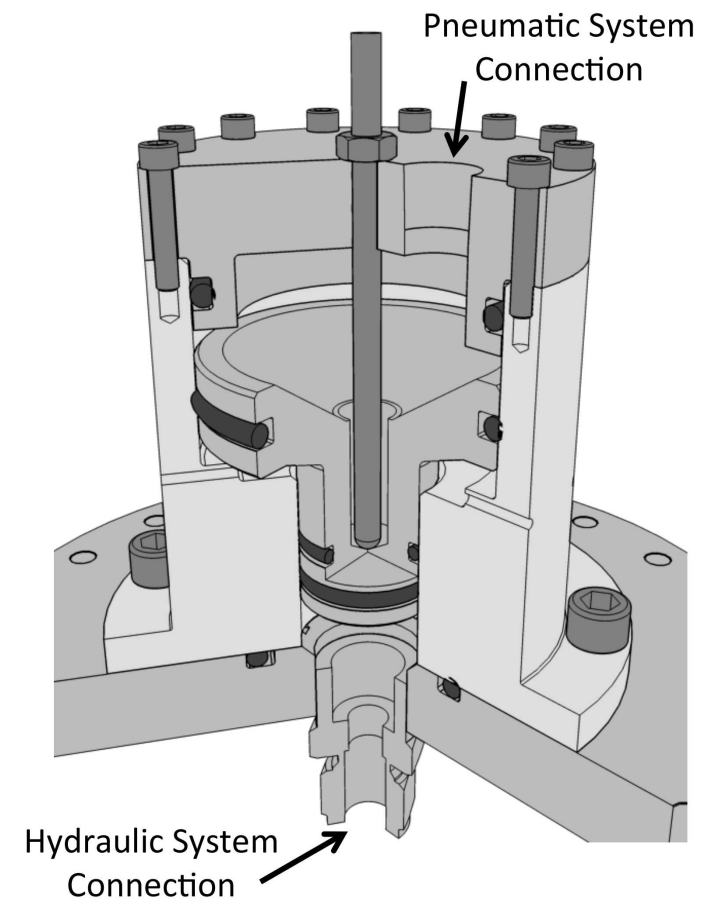

Figure 7: Detail cut-away of the pressure transfer piston and cylinder assembly providing the interface between the pneumatic and hydraulic systems. The lower volume belongs to the hydraulic system, the upper volume is part of the pneumatic system. 
directions is limited by flanges. This helps to prevent the complete vaporization of the superheated fluid in case of an event that proceeds undetected by the video cameras and the computer system.

An accumulator with an adjustable pneumatic charge is used for thermal expansion compensations and when significant temperature excursions are needed during filling operations. Once the detector is filled and has reached its operating temperature, the accumulator is valved off and put in standby. The hydraulic system pressure is monitored by a fast response $(<1 \mathrm{~ms})$ pressure transducer [18]. It is continuously monitored to ensure the correct superheat and recompression pressure. Its value is also recorded for each detected event and event triggering is possible on the pressure signal. This means that as pressure rises due to bubble growth, the recompression cycle can be initiated without the need of the optical signal from the video cameras.

A proper filling of the system with the various liquids is critical. Trapped gases in the hydraulic system result in poor pressure response. Small amounts of the active fluid in contact with rough surfaces outside the glass vessel cause pressure control problems as well as rapid boiling during decompression. A typical filling procedure involves the evacuation of the entire hydraulic system to remove all trapped gases. Distilled and degassed water is then allowed into the system through a filter until the entire volume of hydraulic system is filled with water. The accumulator is then used to increase the pressure to slightly below the vapor pressure of the active fluid at room temperature and the hydraulic system is chilled. Once this is complete, gaseous $\mathrm{C}_{4} \mathrm{~F}_{10}$ is slowly allowed into the glass pressure vessel through a filling stinger (see Fig. 6). The gas condenses as it travels down through the stinger and sinks to the bottom of the glass pressure vessel. The active fluid then displaces the buffer fluid in the bottom of the glass vessel, and the change in fluid volume is taken up by the piston accumulator. Visual inspection allows for the determination of the success of the filling process. Once filling is complete, a sliding seal and a ball valve facilitate the removal of the filling stinger. The accumulator is valved out of the hydraulic system and proper pressure response is verified. The accumulator can 


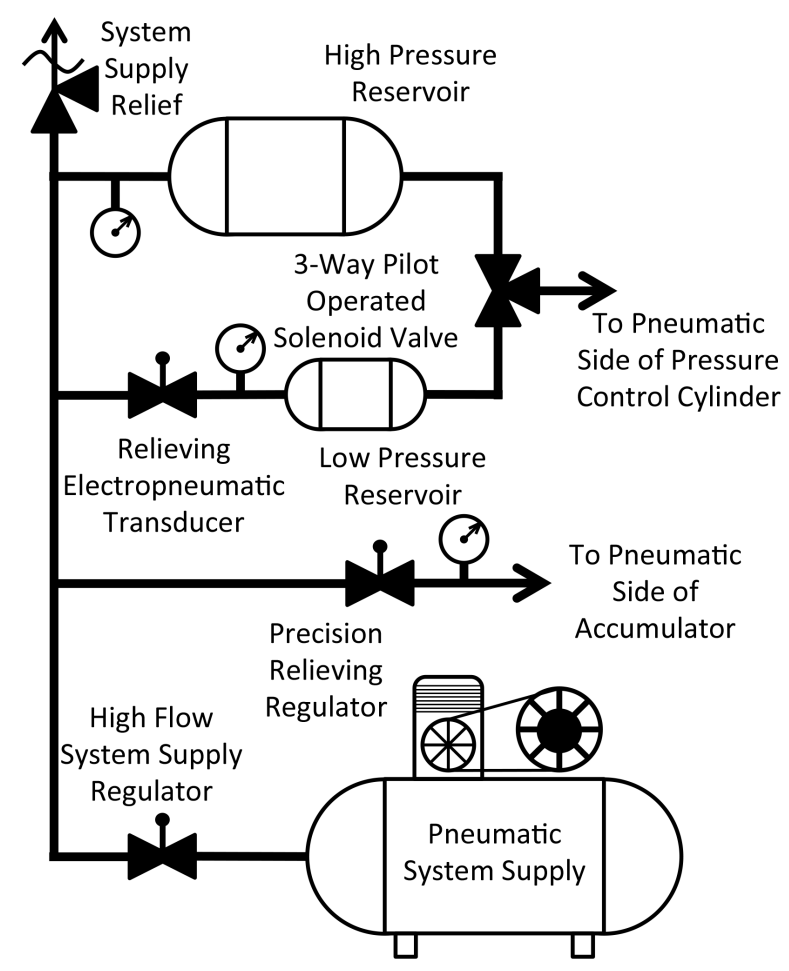

Figure 8: Schematic of the pneumatic system.

be valved back in to the system and the temperature can be increased to the operating temperature to prepare the system for operation.

\subsection{Pneumatic pressure control and temperature monitoring}

The pressure in the hydraulic system is controlled by the pneumatic system as shown in Fig. 8. A fast response $(10 \mathrm{~ms})$ high flow rate, three-way pilotoperated pneumatic solenoid valve [19] is directly connected to the cylinder head of the pressure control cylinder. This valve is controlled by a signal from the DAQ\&C computer, which allows for rapid pressure changes from superheat to compressed conditions. The valve is also connected to two pneumatic tanks which act as reservoirs. The main compression tank is fed directly from a compressed air source and regulated to achieve the proper compression pressure. A connection from this supply is routed to a precision self-relieving regulator, 
which feeds the superheat pressure tank. This regulator is an electropneumatic transducer [20], which allows for remote control of its output pressure. This enables fine control of superheat pressure without the need to access the device.

Since the temperature of the system has to be stable to within $1^{\circ} \mathrm{C}$ the interior of the containment vessel is thermally insulated. Several resistance temperature detectors (RTDs) are mounted on the glass vessel to monitor the temperature of the superheated liquid. The signals from the RTDs are fed into temperature controllers which display and control the temperature in the chamber by activating a small heater installed near the bottom of the safety vessel. Thermal gradients across the active volume are smaller than $0.5^{\circ} \mathrm{C}$.

\subsection{CMOS cameras, lighting and data acquisition}

The containment vessel has two viewing ports located at $\pm 45^{\circ}$ with respect to the incident $\gamma$-ray beam. Two $100 \mathrm{~Hz}$ high-sensitivity CMOS area scan cameras [21] are continuously monitoring the active volume of the glass vessel. To increase contrast and sensitivity, a high intensity diffuse LED backlight is mounted opposite to each camera. One of the cameras acts as master and one as slave. The DAQ\&C computer continuously subtracts each new frame provided by the master camera from the previous frame and evaluates the difference. It takes about $5 \mathrm{~ms}$ to analyze the frames, which allows us to take full advantage of the speed of the camera (10 ms per frame). Once a difference above a certain threshold has been detected, the system is triggered by sending a signal to the control system to repressurize. A time stamp is assigned to this event and a sequence of 10 consecutive frames from the master camera as well as one frame from the slave camera is written to disk. This provides spatial information about the event within the vessel. Pressure and temperature condition of the bubble chamber at the time of the event are also recorded. As an example Fig. 9 exhibits the sequence of 10 pictures from the master camera which show the growth and the recompression of one bubble event. The bubble is found to grow for the first $40-50 \mathrm{~ms}$, which is the time needed to (1) recognize a difference in two subsequent frames, (2) send a signal to a fast relay which 


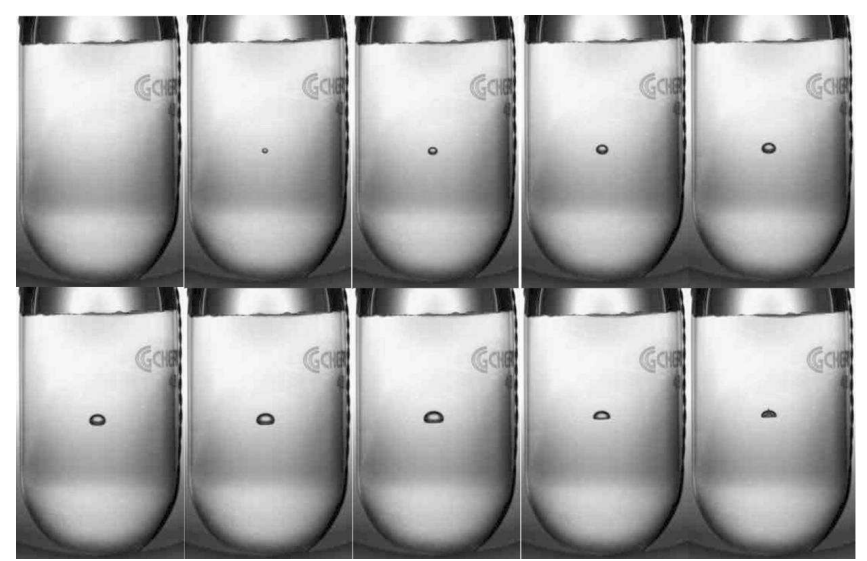

Figure 9: Series of 10 pictures taken by the CMOS camera showing the development of a bubble generated in superheated $\mathrm{C}_{4} \mathrm{~F}_{10}$ via the ${ }^{19} \mathrm{~F}(\gamma, \alpha){ }^{15} \mathrm{~N}$ reaction. The time difference between individual pictures is $10 \mathrm{~ms}$.

energizes the pneumatic valve, and (3) pressurize the pneumatic volume of the pressure control cylinder. This initiates a pressure increase sufficient to quickly liquify the bubble. After an (adjustable) time difference, typically of 1 to $5 \mathrm{~s}$, which is the main component to the dead time (see figure 5 for a compression of $2.1 \mathrm{~s}$ ), the DAQ\&C system decompresses the bubble chamber again so that it is ready for the next event.

Given the small growth rate of a bubble during the first $10 \mathrm{~ms}$ after nucleation, the bubble chamber is able to resolve the site of bubble formation with a resolution of the order of the resolving power of the video camera. This is well below $1 \mathrm{~mm}$, i.e. smaller than the size of the $\gamma$-ray beam which is defined by a $10 \mathrm{~mm}$ collimator.

\section{Experimental Details}

\subsection{Production of the $\gamma$-ray beam}

Linear or circular polarized $\gamma$-rays with energies ranging from 5 to $10 \mathrm{MeV}$ are produced at the High Intensity $\gamma$-ray Source $(\mathrm{HI} \gamma \mathrm{S})$ at Duke University [22]. A full description of this facility can be found elsewhere and only a few details will be discussed here. The $\gamma$-rays are produced through inverse Compton scattering of a two-bunch electron beam circulating in an electron storage ring 
with the photons from a high-power free electron laser (FEL) beam. Each electron bunch had different charges: a big one to induce the FEL $(\sim 40 \mathrm{~mA})$ and a small one $(\sim 1 \mathrm{~mA})$ to produce the $\gamma$-ray beam by inverse Compton scattering. This scheme helped reducing the bandwidth of the $\gamma$-ray beam profile.

The energy of the electrons was typically $400 \mathrm{MeV}$ with a $532 \mathrm{~nm}$ wavelength

of the laser light. With this technique intensities up to about $10^{8} \mathrm{\gamma} / \mathrm{s}$ can be achieved. The $\gamma$-ray beam was collimated to a diameter of $10 \mathrm{~mm}$ with a series of oxygen-free $\mathrm{Cu}$ collimators. The $(\gamma, \mathrm{n})$ thresholds of ${ }^{63,65} \mathrm{Cu}$ are at $10.85 \mathrm{MeV}$ and $9.90 \mathrm{MeV}$, respectively, preventing the production of neutrons which, as discussed earlier, can be a source of background in the experiment. For the same reason the entrance window of the safety vessel of the bubble chamber consisted of $3 \mathrm{~mm}$ thick Al flanges. At the low $\gamma$-ray energies used in this experiment contributions from $(\gamma, \mathrm{n})$ reactions on other isotopes ( $\mathrm{such}$ as ${ }^{57} \mathrm{Fe}$ and ${ }^{29} \mathrm{Si}$ ) do not result in any neutron background that trigger the bubble chamber. As will be discussed later, neutrons can be produced through collisions of the electron beam with the residual gas in the storage ring. The flux of the incident $\gamma$-ray beam was controlled by inserting a set of $\mathrm{Cu}$ attenuators into the beam path. Typical beam intensities used in the experiment ranged from $2 \times 10^{3}$ to $5 \times 10^{6}$ $\gamma / \mathrm{s}$

\subsection{Beam profile}

Two $\gamma$-ray spectra measured with a $123 \%$ efficiency HPGe $\gamma$-ray detector (detection efficiency at $1.33 \mathrm{MeV}$ relative to that of a standard 3-in.-diameter, 3-in.-long $\mathrm{NaI}(\mathrm{Tl})$ scintillator) placed $\sim 173 \mathrm{~cm}$ downstream after the bubble chamber are shown in Fig. 10. The count rate in the detector was kept under $50 \mathrm{kHz}$ by attenuating the flux with $10 \mathrm{~cm}$ thick $\mathrm{Al}$ slab or a $23.6 \mathrm{~cm}$ thick $\mathrm{Cu}$ absorber placed between the bubble chamber and the $\gamma$-ray detector.

The measured spectrum was corrected for the background produced by the $\gamma$-ray beam scattered from the exit window of the bubble chamber and the $\mathrm{Al}$ or $\mathrm{Cu}$ absorber and also corrected for the detector response of the HPGe detector using GEANT [24] simulations. The full energy peak detection efficiency 


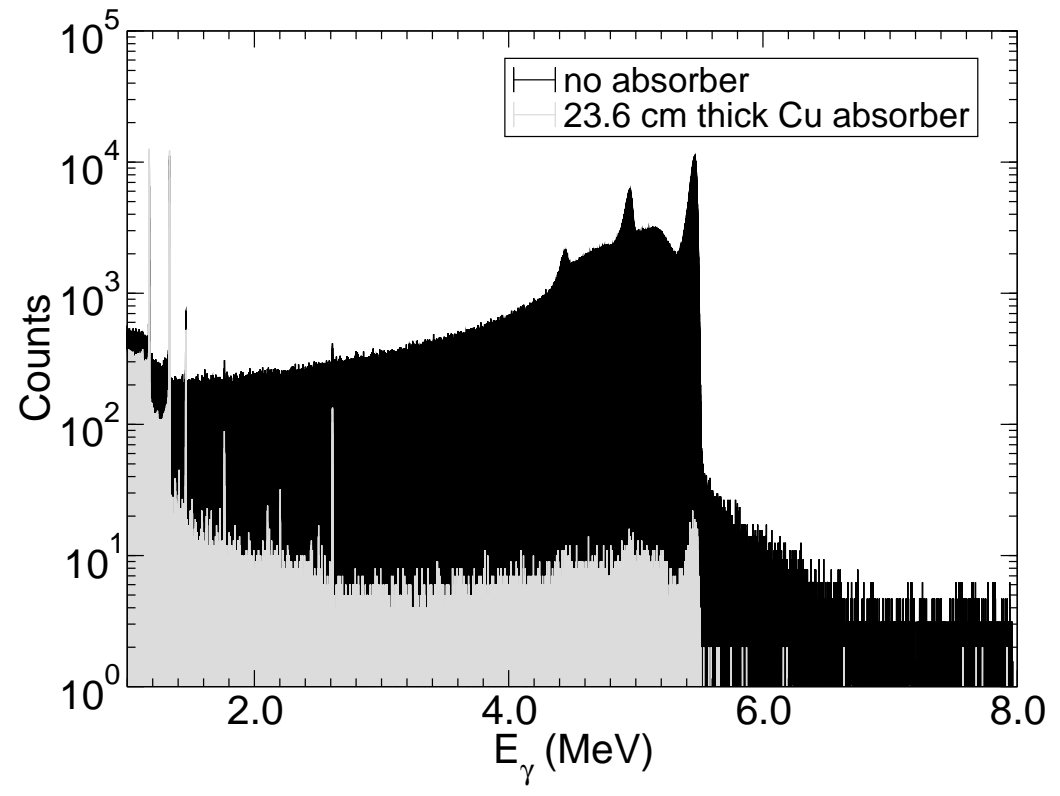

Figure 10: $\gamma$-ray spectra measured with a $123 \%$ HPGe detector placed downstream of the bubble chamber. Inverse Compton Scattering $\gamma$ rays produced by the $\mathrm{HI} \gamma \mathrm{S}$ facility have a centroid energy of $\mathrm{E}_{\gamma}=5.44 \mathrm{MeV}$. The spectra show the total count numbers during 39 minutes of beam measurement. The spectrum in black was taken with a $23.6 \mathrm{~cm}$ thick $\mathrm{Cu}$ absorber between the bubble chamber and the HPGe detector, while the spectrum in gray was acquired with no attenuator in place. Energy calibration for the spectra was performed by placing a ${ }^{60} \mathrm{Co}$ source next to the HPGe detector. The flux of $\gamma$-rays incident on the target was kept constant at $2.8 \times 10^{3} \mathrm{\gamma} / \mathrm{s}$ between both runs. Here, the peaks at $5.44 \mathrm{MeV}$ correspond to the photopeaks, while those around $4.93 \mathrm{MeV}$ are the first escape peak, and those at $4.42 \mathrm{MeV}$ correspond to the second escape peak. 


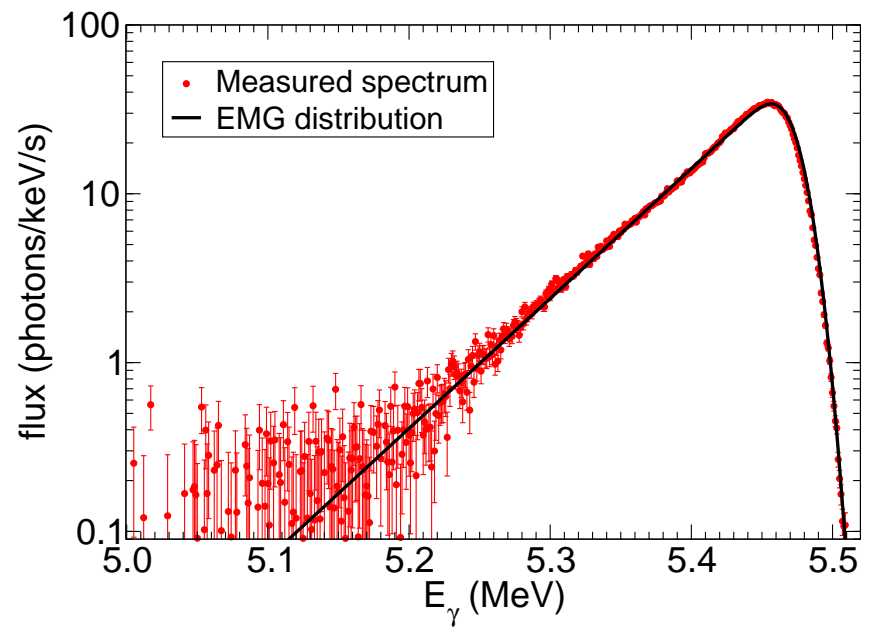

Figure 11: Reconstruction of the incident $\gamma$-ray beam at $\mathrm{E}_{\gamma}=5.44 \mathrm{MeV}$ obtained from a Monte Carlo simulation. The solid line represents the parameterization of the Monte Carlo data. The error bars contain statistical contributions only. An extra 3\% error bar for systematic effects needs to be added to the data.

was simulated using the energy spatial correlation of the $\gamma$-rays in the beam [25] unique for each measurement. The $\gamma$-ray flux was obtained by dividing the background corrected spectrum by the efficiency and the live time of the measurement.

An Exponentially Modified Gaussian (EMG) distribution with the form

$$
\begin{aligned}
f(E) & =\frac{a c \sqrt{2 \pi}}{2 d} \exp \left(\frac{b-E}{d}\right) \\
& +\frac{c^{2}}{2 d^{2}}\left[\frac{d}{|d|}-\operatorname{erf}\left(\frac{b-E}{\sqrt{2} c}+\frac{c}{\sqrt{2} d}\right)\right]
\end{aligned}
$$

was assumed to obtain the measured flux.

Here a, b, c, and d are the fitting parameters, and $\mathrm{E}$ is the $\gamma$-ray energy. A comparison of the experimental and the Monte Carlo simulated flux is shown in Fig. 11 with the solid line representing the EMG distribution.

\section{Experimental results}

For a proof-of-principle study of this detector system we have measured the ${ }^{15} \mathrm{~N}(\alpha, \gamma){ }^{19} \mathrm{~F}$ reaction via photodissociation of ${ }^{19} \mathrm{~F}$, i.e. through the ${ }^{19} \mathrm{~F}(\gamma, \alpha){ }^{15} \mathrm{~N}$ 
reaction [26]. Resonance parameters for the $(\alpha, \gamma)$ reaction have been measured previously [27]. Using these values in a Breit-Wigner model we obtain a predicted spectrum shown in Fig. 12 plotted as function of the excitation energy in ${ }^{19} \mathrm{~F}$ with cross sections ranging from $100 \mathrm{pb}$ to $30 \mu \mathrm{b}$. In our measurement with the bubble chamber we have studied this excitation energy region with $\gamma$-rays from 5-6 MeV. The beam intensities ranged from $2 \times 10^{3}$ to $5 \times 10^{6} \mathrm{\gamma} / \mathrm{s}$ depending on the cross section. The typical running time for one energy was about 1 hour. From the information of the two CMOS cameras located $\pm 45^{\circ}$ with respect to the incident beam one can calculate the shape of the intersection between the beam and the glass vessel. This is shown in Fig. 13, where the location of bubbles measured during one run appear looking along and perpendicular to the $\gamma$-ray beam. No corrections for refraction in the walls of the glass vessel have been made as the position shifts observed were minimal with and without the superheated liquid. The dimensions of the fiducial area are in good agreement with the expectation. The events outside the fiducial volume are present even without a $\gamma$ beam and are caused by ${ }^{12} \mathrm{C}$ and ${ }^{19} \mathrm{~F}$ recoils after elastic scattering of cosmic ray induced neutrons. The neutron-induced background rate in the fiducial volume is about $1 \times 10^{-3}$ events/s. The number of bubbles, $\mathrm{N}_{\text {counts }}$, measured at a given energy and corrected for background was then used to calculate the reaction yield, given by

$$
\text { Yield }=\left(\frac{N_{\text {counts }}}{\phi_{\gamma}}\right) \frac{1}{\Delta t-t_{\text {dead }} * N_{\text {bubble }}} .
$$

Here $\phi_{\gamma}$ is the energy integrated flux of $\gamma$-rays hitting the target (see Fig. 10), $\Delta \mathrm{t}$ the running time, $\mathrm{t}_{\text {dead }}$ the dead time of the detector discussed earlier $\left(\mathrm{t}_{\text {dead }}=2 \mathrm{~s}\right)$ and $\mathrm{N}_{\text {bubble }}$ the number of bubbles observed in the whole active volume of the detector.

The cross section of the ${ }^{19} \mathrm{~F}(\gamma, \alpha){ }^{15} \mathrm{~N}$ reaction was then calculated from the reaction yield in equation 5 by

$$
\sigma(\gamma, \alpha)=\frac{Y i e l d}{L} \frac{1}{\nu \rho} \frac{N_{A}}{A},
$$


where $\mathrm{L}$ is the target thickness $(\mathrm{L}=3 \mathrm{~cm}), \nu$ is the number of fluorine atoms per $\mathrm{C}_{4} \mathrm{~F}_{10}$ molecule $(\nu=10), \rho$ is the density of the liquid at superheated conditions $\left(\rho=1.45 \mathrm{~g} / \mathrm{cm}^{3}\right), \mathrm{N}_{A}$ is Avogadro's number, and $\mathrm{A}$ is the molecular weight of $\mathrm{C}_{4} \mathrm{~F}_{10}$ in a.m.u. $(\mathrm{A}=238)$. The cross section of the ${ }^{19} \mathrm{~F}(\gamma, \alpha)^{15} \mathrm{~N}$ reaction was then used to calculate $\sigma\left({ }^{15} \mathrm{~N}(\alpha, \gamma){ }^{19} \mathrm{~F}\right)$ with equation 1 . The results of the cross sections assuming a detection efficiency $\epsilon=1$ are shown as the points in Fig. 14 with the appropriate uncertainties in $\mathrm{E}$ and $\sigma$. The error bar for the cross section corresponds to the statistical uncertainty, while the error bar for the energy was obtained from the FWHM of the $\gamma$-ray beam as modeled in Fig. 11 and converted to $\mathrm{E}_{l a b}$ in the ${ }^{15} \mathrm{~N}(\alpha, \gamma){ }^{19} \mathrm{~F}$ reference system.

The cross sections range from about $10 \mu \mathrm{b}$ at the peak of the resonance to about $3 \mathrm{nb}$ at the lowest energy. At the highest cross section the beam intensity had to be reduced to about $2 \times 10^{3} \gamma / \mathrm{s}$, confirming the high luminosity of this detector system. The solid line corresponds to the excitation function folded with the resolution function of the beam as described in Fig. 11. As can be seen an excellent agreement between the experimental data and the theoretical prediction is observed, so the assumption of $\epsilon=1$ for the detection efficiency of the reaction products is also confirmed. This is in agreement with the results presented in Ref. [7].

\subsection{Beam-induced background}

As can be seen from Fig. 14, the experimental cross sections at the lowest energies saturate at $\sim 3 \mathrm{nb}$, overestimating the value predicted by the BreitWigner distribution (see Fig. 12). The count rate measured at these energies contain a contribution from a beam induced background source that has not been accounted for so far. These background signals cannot be distinguished from the signal of interest from ${ }^{19} \mathrm{~F}(\gamma, \alpha){ }^{15} \mathrm{~N}$, as they appear in the same spatial region when the beam is incident on the target. They are caused by a Bremsstrahlung radiation component produced by the electrons and the residual gas in the beam line. While small in comparison with the main $\gamma$-ray beam component, these $\gamma$-rays have energies ranging from the electron beam energy 


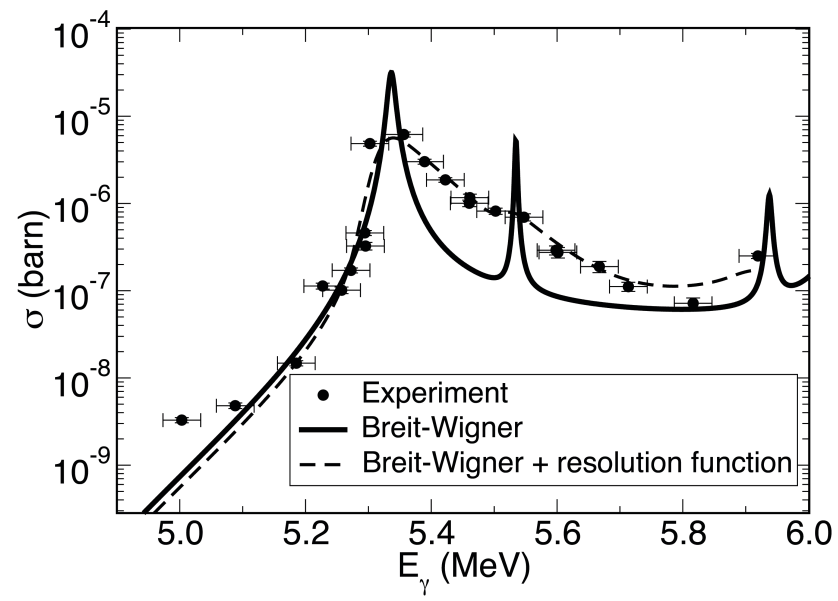

Figure 12: Cross section for the ${ }^{15} \mathrm{~N}(\alpha, \gamma){ }^{19} \mathrm{~F}$ reaction computed with a Breit-Wigner model using parameters from [27]. In solid black, the cross section is presented, while in dashed black, the curve folded with the resolution function (see Fig.11) of the beam is shown.

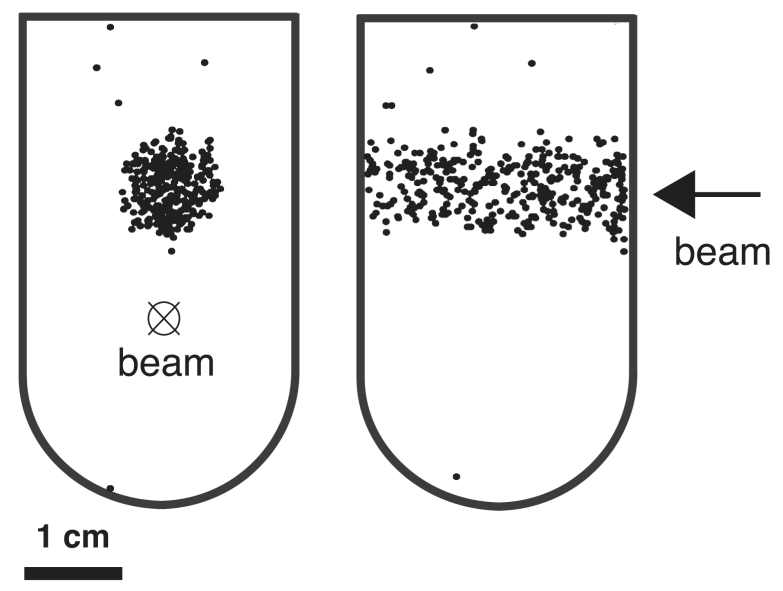

Figure 13: Sites of nucleation for a beam-on-target run. The profile of the $\gamma$-ray beam can be clearly observed. Some background events are also seen. 


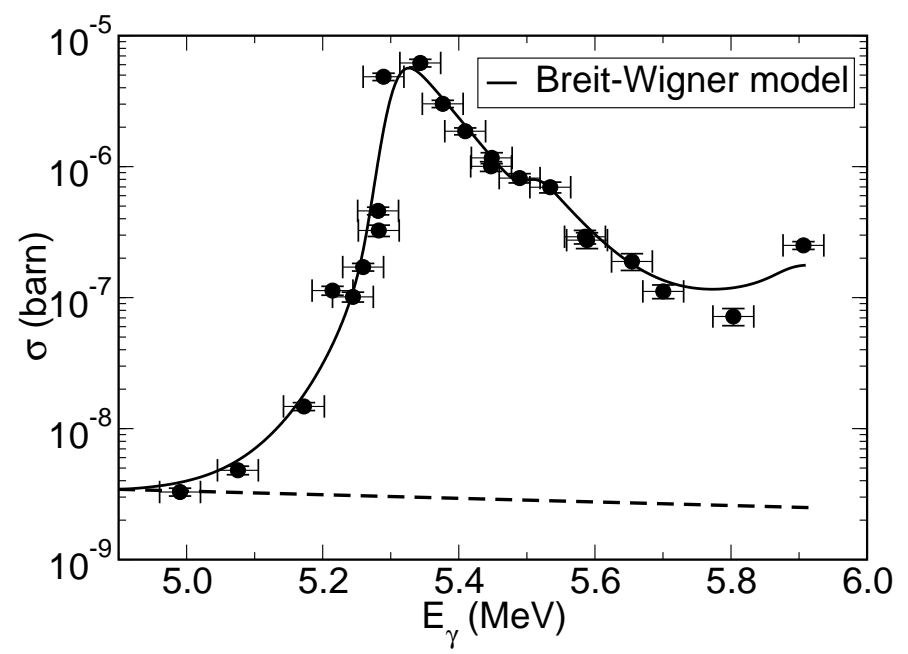

Figure 14: Cross sections calculated with a Breit-Wigner model with resolution function and corrected with a Bremsstrahlung background component. The data points were obtained experimentally and are in good agreement with the model. The dashed curve represents the background while the solid line is the sum of the dashed line of figure 12 and the Bremsstrahlung background.

(some hundreds of $\mathrm{MeV}$ ) down to zero. Thus even a small amount of high-energy Bremsstrahlung $\gamma$-rays can dominate the yield at the lowest energies.

In order to estimate the flux from this background source we assume electrons circulating in a storage ring in which the vacuum has a typical value of $2 \times 10^{-10}$ Torr. The section of the beam line in the ring where the electrons move in the direction of the bubble chamber has a length of $35 \mathrm{~m}$. Assuming a beam energy of $400 \mathrm{MeV}$, an electron beam current of $40 \mathrm{~mA}, \mathrm{Z}=10$ residual gas [30], a $3 \mathrm{~cm}$ thick $\mathrm{C}_{4} \mathrm{~F}_{10}$ target with $(\gamma, \mathrm{n})$ cross sections of $15 \mathrm{mb}$ between 15$30 \mathrm{MeV}$ and $0.5 \mathrm{mb}$ elsewhere the count rate for this Bremsstrahlung induced background source would be about 0.1 counts per second, which is in good agreement with the count rate values measured at the lowest cross sections in our experiments. Converting this background yield into cross sections and plotting it in the ${ }^{15} \mathrm{~N}(\alpha, \gamma){ }^{19} \mathrm{~F}$ plot gives the dashed line in Fig. 14. Adding this contribution to the folded Breit-Wigner distribution results in an excellent agreement with the experimental data.

There are two possible ways of reducing this background contribution: the 
first includes the suppression of the Bremsstrahlung radiation by rearranging the accelerator beamlines at the region where the laser-electron collision takes place. The electron beam length in the direction of the $\gamma$ rays could be reduced by bending their path with a magnet right before the collision point. The second possibility includes the separation of neutron-induced events from the heavy ion recoils by using the sound produced by the nucleation events. This technique has been successfully used in superheated liquid devices built for dark matter detection. The different spectral charactersitics of the sound produced by these two types of events allow for their separation [28].

\subsection{Other backgrounds and systematic uncertainties}

Contributions to the count rate that are accounted for as backgrounds and are unrelated to the $\gamma$-ray beam or its production process include cosmic ray induced neutrons and muons, radioactivity in the vessel from the chemical makeup of the glass, and neutrons photodisintegrated with $\gamma$-rays produced by the experimental room walls. All these backgrounds were measured by operating the bubble chamber without an electron beam in the accelerator ring. Cosmic ray induced events will appear evenly distributed over the whole volume of the superheated liquid. They can be reduced by passive shielding around the bubble chamber. Radioactivity from the glass only triggers nucleation on the superheated liquid in close contact with the vessel walls.

These backgrounds can be identified by using the good spatial resolution capabilities of the bubble chamber. By knowing the fiducial region where the $\gamma$-ray beam irradiates the superheated liquid, the majority of these background events can be suppressed. It is only those produced in the fiducial region that need to be accounted for by doing background runs without beam in the accelerator. In these tests, the average time between consecutive nucleation events was of two minutes, while the typical count rate of the detector when $\gamma$-ray irradiated it was of the order of $0.1 \mathrm{~Hz}$. This results in an average $8.3 \%$ contribution to the count rate coming from these sources of background combined. While some bubbles were observed next to the glass walls (one or two per hour 
of operation), the contribution to the whole count rate from these was insignificant. These nucleation sites could be associated with $\alpha$-particle decays from radioactive nuclei in the glass.

An additional background contribution that was studied in this work was that originating from photo-induced neutrons produced upstream in the accelerator by the interaction of $\gamma$-rays with beam line components. We measured this contribution by moving the bubble chamber to the side out of the $\gamma$ ray beam path and determining a count rate. These bubbles were distributed evenly in the superheated liquid volume and account for $8 \%$ of the count rate measured in runs with the $\gamma$-ray beam impinging on the bubble chamber.

Other systematic uncertainties come from the determination of the beam intensity, which ranged from $2 \times 10^{3}$ to $3 \times 10^{6} \mathrm{\gamma} / \mathrm{s}$, with an error bar under $5 \%$, as discussed in [29]. The dead time (see figure 5), determined to be 2.1 seconds, had a systematic error in the range from $\pm 2 \%$ up to $\pm 15 \%$ for measurements at the highest count rate achieved. The length of the liquid target irradiated by the beam was determined to be $3.0 \pm 0.1 \mathrm{~cm}$. The uncertainty was mainly determined by the position of the $\gamma$-ray beam with respect to the center of the target. This effect contributed a $3 \%$ systematic error in the determination of the measured cross sections. The detection efficiency (discussed above) contributes a systematic uncertainty that is negligible compared to other systematic effects.

\section{Summary}

The long life of stars can be understood in the context of the strong Coulomb barriers which prevent nuclei from fusing. It is this same reason that complicates measurements of very small reaction cross sections in the laboratory. Therefore, new state-of-the-art detectors have to be developed to deal with the extremely low count rates. Since bubble chambers allow us to use liquid target material the density is much higher when compared to gas targets which is advantageous for measuring the small cross sections of time-inverse $(\alpha, \gamma)$ reactions. The bubble chamber described in this paper is one of the possible approaches. Since these 
detectors are practically insensitive to $\gamma$-rays only the charged particle reaction products are detected in the active liquid.

We have constructed a device for performing the measurements and established the technique. Future facilities for producing $\gamma$-rays using inverse Compton Scattering will benefit from results obtained in this work. In particular, upcoming facilities using external lasers that do not have significant residual gas in the path of the electron beam and in the same direction of the $\gamma$ ray beam will allow measuring the low cross sections relevant to nuclear astrophysics as high energy Bremsstrahlung radiation will be significantly reduced.

This feature is required regardless of the particle identification method or kinematic information obtained by a detector trying to measure these small cross sections.

\section{Acknowledgement}

This work was supported by the US Department of Energy, Office of Nuclear Physics, under contract No. DE-AC02-06CH11357. We want to thank the operating group at $\mathrm{HI} \gamma \mathrm{S}$ for providing the high quality beams. Discussions with Prof. Ying K. Wu and Dr. Stepan F. Mikhailov about the source of the background are appreciated. We also thank Sebastian Rehm for writing the LabView computer code used in the acquisition of the experimental data.

\section{References}

[1] J. M. Blatt and V. F. Weisskopf, Theoretical Nuclear Physics (SpringerVerlag, 1979).

[2] M. M. Szydagis, Dark Matter limits from a $15 \mathrm{~kg}$ windowless bubble chamber, Ph.D. thesis, University of Chicago, 2011.

[3] W. J. Bolte et al., Nucl. Instrum. Meth. A 577 (2007) 569.

[4] S. E. Woosley, Astrophys. J. 330 (1988) 218.

[5] D. A. Glaser, Nuovo Cimento 11, Suppl. 2 (1954) 361. 
[6] D.V. Jordan et al., Appl. Radiat. Isotopes 63, (2005), 645.

[7] V. Zacek, Nuovo Cimento A 107 (1994) 291.

[8] T. A. Girard et al., Phys. Lett. B 621, 233 (2005).

[9] E. Ramberg et al., Nucl. Instrum. Meth. A 623 (2010) 454.

[10] Harper, M. J., Nucl. Sci. Eng. 114 (1993) 118 .

[11] L. Gunther, Am, J, Phys. 71 (2003) 351.

[12] M. J. Uline and D. S. Corti, Phys. Rev. Lett. 99, (2007) 076102.

[13] F. Seitz, Phys. Fluids Vol.1, (1958) 2-13.

[14] Lemmon, E.W., Huber, M.L., McLinden, M.O. NIST Standard Reference Database 23: Reference Fluid Thermodynamic and Transport PropertiesREFPROP, Version 9.1, National Institute of Standards and Technology, Standard Reference Data Program, Gaithersburg, 2013.

[15] S. Archambault et al., New J. Phys. 13 (2011) 043006.

[16] Perfluorobutane, FluoroMed, L.P, www.fluoromed.com

[17] CG-1880-24, Chemglass Life Sciences, www.chemglass.com

[18] MM Series Pressure Transducer, Omega Engineering, Inc., www.omega.com

[19] Series 57 Valves, MAC Valves, Inc., www.macvalves.com

[20] Type 500X Electropneumatic Transducer, ControlAir, Inc., www.controlair.com

[21] A600 Series Area Scan Camera, Basler, Inc., www.baslerweb.com

[22] H. R. Weller et al., Research opportunities at the upgraded HI $\gamma \mathrm{S}$ facility. Prog. Part. Nucl. Phys., 62:257, 2009. 
[23] W. Press et al., Numerical Recipes in C, Cambridge University Press, 1992.

[24] R. Brun et al., GEANT 3.21 DD/EE/94-1 (CERN, Geneva, 1994).

[25] Sun, C. et al., Phys. Rev. ST 12 (2009) 062801.

[26] C. Ugalde et al., Phys. Lett. B 719 (2013) 74.

[27] S. Wilmes et al., Phys. Rev. C 66 (2002) 065802.

[28] F. Aubin et al., New J. Phys. 10 (2008) 103017

[29] S. Carson, et al., Nucl. Instr. Meth. A 618 (13) (2010) 190.

[30] E. C. Schreiber, "Measurement of a high-intensity gamma-ray beam and the analyzing power for ${ }^{2} \mathrm{H}(\gamma, \mathrm{n}) \mathrm{p}$ near threshold", Ph.D. thesis, Duke University, 2000. 